\title{
Biotechnological

\section{Effect of gel porosity and stiffness on culture of HepG2 cells encapsulated in gelatin methacrylate hydrogels}

\author{
Mansi K. Aparnathi and Jagdish S. Patel* \\ P. D. Patel Institute of Applied Science, Charotar University of Science and Technology, Changa-388421, \\ Gujarat, India
}

\begin{abstract}
Owing to in vivo applications, use of biodegradable three dimensional matrices to form implantable tissue constructs has increased in recent times. Gelatin methacrylate gel (GelMA) is one such versatile matrix compatible for cell culture and has potential of in vivo implantation. Physical and mechanical properties of these hydrogels are extremely crucial in modulating their rigidity, biodegradability and cellular compatibility. The present study involves testing stiffness and porosity of GelMA at high and low methacrylation degrees and at different concentrations of prepolymer and itseffect on viability and proliferation of HepG2 cells cultured encapsulated in GelMA. Stiffness was found to be directly proportional whereas porosity was found to be inversely related to degree of methacrylation and concentration of GelMA pre-polymer. Softer gel with greater porosity was found to be favorable for proliferation of cells encapsulated in the gel. In order to achieve stable implantable construct, it is important to fine tune its physical properties such as stiffness and porosity since both these parameters are responsible for governing culture of cells encapsulated within the gel matrix and the present study will be helpful in modulating these properties of GelMA as per the intended application.
\end{abstract}

\section{KEY WORDS: GELMA, PHYSICAL PROPERTIES, TMSPMA COATING}

\section{INTRODUCTION}

Biomaterial tissue constructs that have found applications as in vivo implants are constructed by encapsulating cells in three dimensional matrices. The cells in constructs are either cultured prior to implantation or directly implanted and allowed to grow in vivo post- implantation (Bhatia \&t Chen, 1999).It has been reported that the development of tissue within gel matrix is modulated by surface chemistry of the scaffold, its pore size, structure and mechanical properties, though gold standard of parameters has not been defined yet (Basu, 2004). Hollister et al. have explained the requirement to modulate physical properties of gel matrix so as to

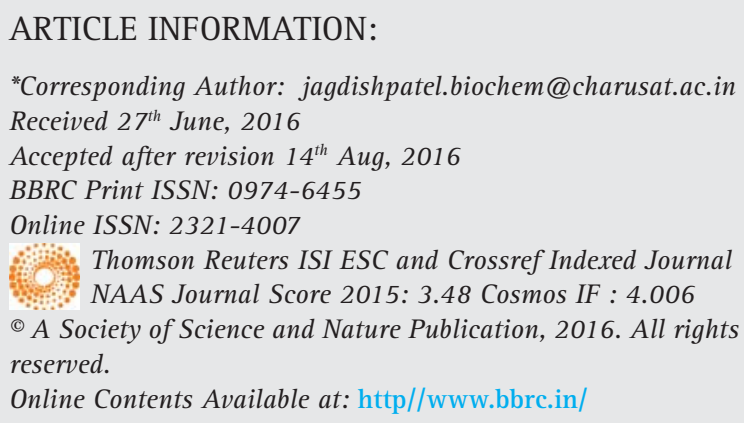


suit the intended application (2002).A biodegradable, architecturally flexible, photocrosslinkable and biocompatible gel has been synthesized from methacrylated gelatin gel GelMA (Van Den Bulcke et al., 2000) and has been exploited for cell culture studies by various groups (Chen et al., 2012; Nichol et al., 2010; Nikkah et al., 2012; Samorezov et al., 2016).

Mechanical properties such as stiffness and rigidity of cell matrix varies with the tissue type, being higher for bones and lower for soft tissues (Tse and Engler, 2010). It is important for tissue construct intended for implantation to mimic the stiffness of the tissue found naturally in the body. Tissue stiffness is important in determining cellular function, and changes in tissue stiffness are commonly associated with fibrosis, cancer and cardiovascular disease (Cretu et al., 2010).Mechanical properties of GelMA can be tuned to desired parameters by altering the methacrylation levels of the gel (Hoch et al., 2012) and percentage of GelMA in pre-polymer used for crosslinking the gel (Chen et al., 2012).

Porosity of matrix is extremely important parameter which determines the degree of solute diffusion, surface properties, mechanical properties and surface mobility (Peppas et al. 2006). Gel with higher porosity is reported to be more suitable for cell proliferation(Hoch et al., 2012). Higher porosity was achieved in calcium alginate/gelatin hydrogels which were shown to possess enhanced compatibility for cell culture (Cuadros et al., 2015). Even the osteogenic differentiation of cells can be modulated by finetuning porosity of hydroxyapatite (Tsurugaet al.,1997). Porosity of GelMA as a function of its swelling ratio has been extensively studied by Nichol et al. (2010). The degree of swelling of gels relies on the porosity of the matrix and the solvent-polymer interaction (Du et al., 2008).

In the present study, mechanical testing was to be carried out in order to determine the compressive modulus of GelMA at varying degrees of methacrylation and pre-polymer concentration. Swelling analysis and SEM imaging of gels for determination of porosity of GelMA were undertaken. Compatibility of GelMA as a matrix for culture of HepG2 cells was determined along with the best degree of methacrylation via live-dead staining and MTT proliferation assay. This appears to be the first study from India, where potential of GelMA as a tissue engineering implant has been explored. The main aim of the present study was to help in fine-tuning the gel properties based on its intended application.

\section{MATERIAL AND METHODS}

\section{SYNTHESIS OF GEIMA}

High and low methacrylated GelMA were synthesized by the protocol described by Nichol et al. (2010). Briefly,
Type-A porcine skin gelatin (Sigma)was dissolved in DPBS (Sigma) yielding $100 \mathrm{ml}$ 10\% gelatin solutionat $60^{\circ} \mathrm{C}$ for $1 \mathrm{hr}$. Methacrylic anhydride (MA) (Sigma) was added drop wise to the gelatin solution and was allowed to react for $3 \mathrm{hrs}$ at $60^{\circ} \mathrm{C} .800 \mu \mathrm{MA}$ was added for the synthesis of low methacrylated gelatin gel $\left(\mathrm{GelMA}_{\text {low }}\right)$ and $8 \mathrm{ml} \mathrm{MA}$ was added for the preparation high methacrylated gelatin gel $\left(\mathrm{GelMA}_{\text {high }}\right)$. Solution was diluted $5-X$ by adding warm DPBS and allowed to mix well at $50^{\circ} \mathrm{C}$ for $1 \mathrm{hr}$. GelMA solution was dialyzed using $12-14$ $\mathrm{kDa}$ cut-off dialysis tubes (HiMedia) against deionized water for 7 days at $50^{\circ} \mathrm{C}$. The dialyzed GelMA solutions were frozen at $-80^{\circ} \mathrm{C}$ for at least 5 days, freeze dried and stored at room temperature until use.

\section{GeIMA pre-polymer}

Freeze-dried GelMA (depending upon the final \% of GelMA required) and 0.5\% photoinitiator, 2-Hydroxy4'-(2-hydroxyethoxy)-2-methylpropiophenone (Sigma) were dissolved in DPBS at $70^{\circ} \mathrm{C}$ to prepare pre-polymer solution of GelMA. GelMA pre-polymer could be filled in desired mold and photocrosslinked by exposing it to $6.7 \mathrm{~mW} / \mathrm{cm}^{2}$ UV light for $1 \mathrm{~min}$ at room temperature.

\section{MECHANICAL TESTING}

Fifty microliters of pre-polymer wasphotocrosslinkedinto cylindrical wells fabricated in PDMS mold. Dimensions of the discs to be used for mechanical testinghave approximately $0.75 \mathrm{~mm}$ diameter and $0.5 \mathrm{~mm}$ height. Samples were detached from the mold and transferred free floating at $37^{\circ} \mathrm{C}$ in DPBS. Immediately prior to testing, the disc was blotted lightly with a wipe and testedon an Instron 5542 mechanical tester. The compressive modulus was determined as the slope of the linear region corresponding with Young's Compressive stress $1 \%-10 \%$.

\section{POROSITY ANALYSIS}

\section{Scanning Electron Microscopy(SEM)}

GelMA sheets were made by crosslinking $30 \mu \mathrm{l}$ of $10 \%$ GelMA $_{\text {high }}$ and GelMA $A_{\text {low }}$ pre-polymer between two glass slides separated by a $1 \mathrm{~mm}$ spacer as demonstrated in schematic Figure 1. Sheets were detached from slide, frozen at $-80^{\circ} \mathrm{C}$ overnight and lyophilized. These sheets were further visualized under FEG-SEM (Nova Nano SEM 450)tocompare porosity of both the gels.

\section{Swelling analysis}

Discs with 5 and $10 \%$ of GelMA $A_{\text {high }}$ and GelMA $A_{\text {low }}$ were prepared and weighed. These discs were allowed to 


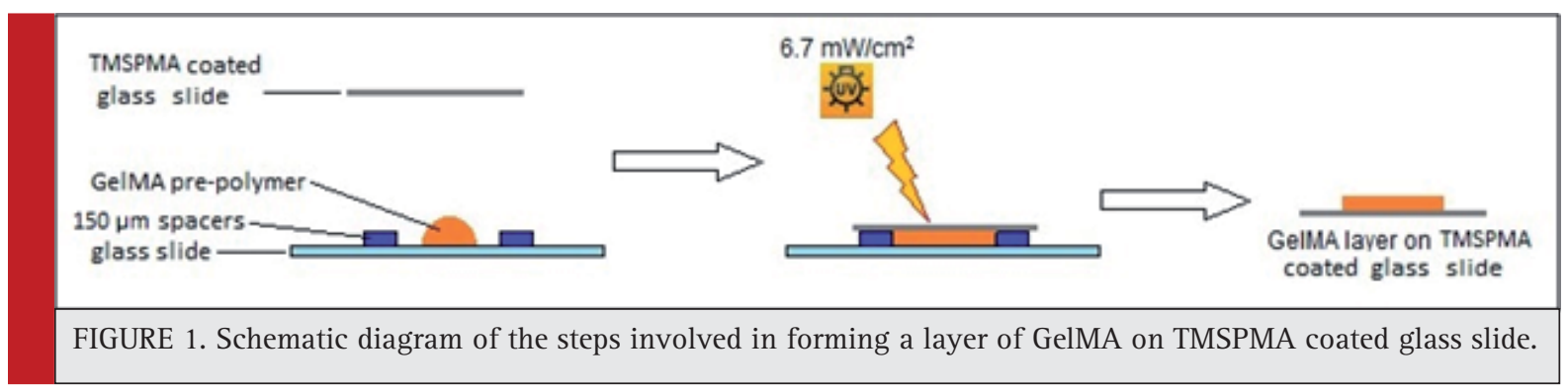

hydrate in DPBS for $24 \mathrm{hrs}$ in humidified $\mathrm{CO}_{2}$ incubator at $37^{\circ} \mathrm{C}$. Discs were removed from PBS, blotted on wipes and weighed. Percentage increase in weight was calculated. Experiment was repeated thrice and discs for each set of experiment was made in triplicates.

\section{TMSPMA COATING OF GLASS SLIDES}

TMSPMA coating is essential for the adherence of GelMA sheets to glass slide. $50 \mathrm{~g}$ of Sodium Hydroxide pellets (NAOH, Sigma) were dissolved in $450 \mathrm{~mL}$ of distilled water. Glass slides stacked in staggered manner were treated with this NAOH solution overnight such that each surface of every slide stays in contact with the solution. Slides were then rinsed and rubbed under distilled water. Slides were then sequentially plunged in five tubes containing reagent alcohol and allowed to air dry. Slides wrapped in aluminium foil were baked at $80^{\circ} \mathrm{C}$ for $1 \mathrm{hr}$. Slides were stacked vertically and 3-4 ml of TMSPMA (3-(Trimethoxysilyl)propyl methacrylate, Sigma) was dripped over the stack with a syringe and allowed to flow over the slide for $30 \mathrm{~min}$. The stack was inverted and again coating was allowed to go on for another $30 \mathrm{~min}$. Beaker was covered with aluminum foil and baked overnight at $80^{\circ} \mathrm{C}$. Slides were again given 5 washes with reagent alcohol and air dried. Slides were wrapped in aluminum foil and baked for $1 \mathrm{hr}$ at $80^{\circ} \mathrm{C}$. The TMSPMA coated glass slides wrapped in aluminum foil were stored at room temperature until use. These slides were cut into $1 \times 1 \mathrm{~cm}$ pieces with a glass cutter prior to use.

\section{ENCAPSULATION AND CELL CULTURE}

A confluent monolayer ofHepG2 cells was trypsinized, spun down, trypsin was removed and pellet was re-suspended in $3 \mathrm{ml}$ Dulbecco's Modified Eagle's Medium (DMEM, Sigma) with 10\% Foetal Bovine Serum (FBS, Gibco). Cells were counted under hemocytometer and aliquots with 1 million cells were made. These aliquots were spun down, supernatant was removed and pellet was re-suspended by gently pipetting pellet into $1 \mathrm{ml}$ 10\% GelMA pre-polymer solution so as to obtain 1 million cells per ml of GelMA.
Cell-GelMA suspension was crosslinked on a TMSPMA coated glass slide as per the schematic diagram (Figure 1). Briefly, $20 \mu \mathrm{l}$ cell suspension was placed between 150 $\mu \mathrm{m}$ spacers attached on a glass slide. $1 \mathrm{x} 1 \mathrm{~cm}$ TMSPMA coated glass slide was inverted on it. This system was exposed to UV for crosslinking GelMA. Cells encapsulated in GelMA layered on TMSPMA coated glass slide was submerged in media and incubated in a humidified $\mathrm{CO}_{2}$ incubator at $37^{\circ} \mathrm{C}$. Media was changed every alternate day. Cells were observed microscopically under Leica DMIL inverted microscope.

\section{VIABILITY AND PROLIFERATION ANALYSIS}

\section{Live dead staining}

Viability of HepG2 cells cultured encapsulated in GelMAhigh and GelMA $\mathrm{I}_{\text {low }}$ was determined by calcein-ethidium homodimer Live-Dead assay (Invitrogen) of construct on day 3 of culture using manufacturer's protocol.

\section{MTT assay}

Proliferation of cultured cells was observed 1, 3 and 5 days after cell seeding. Growth in 10\% GelMA $\mathrm{high}_{\text {was }}$ wampared with growth in 10\% GelMA low $_{\text {. MTT Assay described }}$ by Denizot and Lang method (1986) was employed.

\section{RESULTS AND DISCUSSION}

GelMA hydrogels are emerging biomaterials for biomedical applications, mainly owing to their biocompatibility, architectural flexibility and biodegradability. Crosslinked polymer gels can be fabricated with controlled stiffness, pore size and other physical properties. These controlled parameters can be achieved by varying degree of methacrylate substitution or biopolymer concentration (Van Den Bulcke et al., 2000).

\section{GELMA SYNTHESIS}

High and low methacrylated gelatin gels were synthesized (Figure 2a ct2b, respectively), of which further 5\%, 


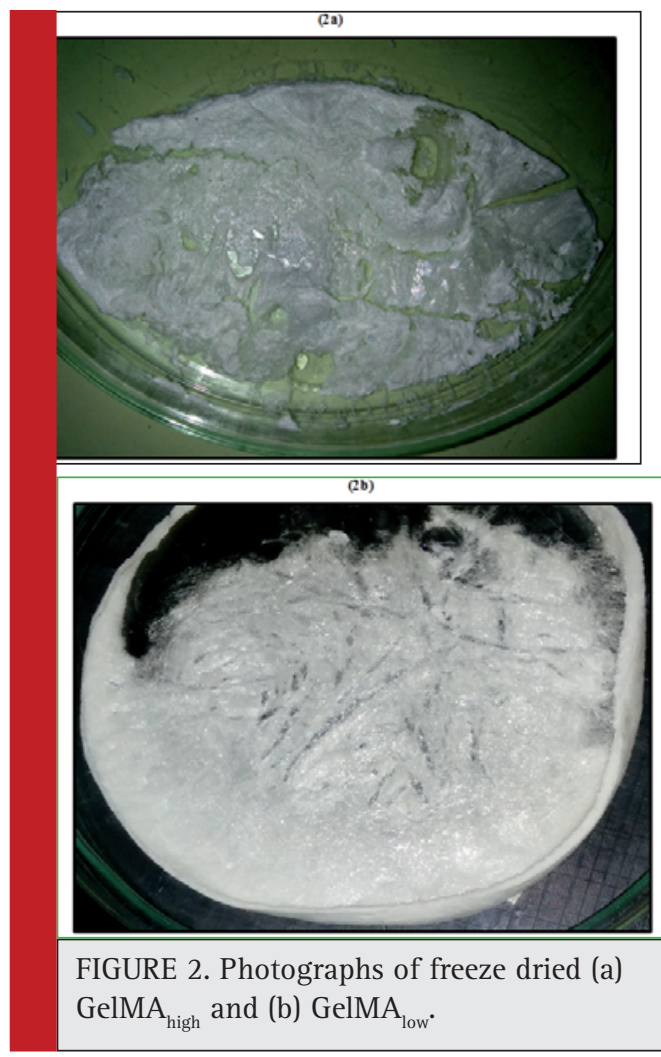

$10 \%$ and 20\% pre-polymers were used for each degree of methacrylation for further study.

\section{MECHANICAL PROPERTIES}

GelMA discs of 5\% and 10\% of GelMA $A_{\text {high }}$ and GelMA were compressed on instron for determining the effect of gel concentration and degree of methacrylation on the mechanical properties of the GelMA (Figure 3). At a given degree of methacrylation, both 5\% and 10\% gels demonstrated a dramatic difference in their compressive modulus. At 10\% concentration, significant difference was observed in compressive modulus of both, high and low methacrylated GelMA. On the contrary, there was no drastic difference in compressive modulus of both the gels at 5\% concentration. No sample failure was reported before the maximum $50 \mathrm{~N}$ load was reached, providing testimony to the elastomeric properties of the GelMA. Chen et al. have demonstrated increase in the compressive modulus of the GelMA with its methacrylation degree (2012). Nichol et al. have reported that increasing the degree of methacrylation increased the stiffness at all the gel concentrations and at a constant degree of methacrylation, increase in the GelMA concentration significantly increased the compressive modulus (2010).

In accordance with their findings, the present study also has shown direct proportionality between degree of methacrylation of GelMA and its compressive modu- lus, which is further proportional to the stiffness of the hydrogel. Moreover, similar proportionality has also been observed between concentration of GelMA and compressive modulus. But, at a given concentration

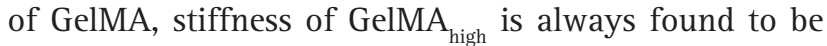
higher than GelMA $\mathrm{low}_{\text {low }}$. In contrast with the study reported by Nichol et al., in present study, the instron analysis of 5\% low methacrylated GelMA was successful, probably on account of different dimension of GelMA disc being used for the test and PDMS mold based fabrication of the disc instead of punching sheets, making the gel easier to handle.

\section{POROSITY ANALYSIS}

\section{SEM}

SEM imaging of the surface of freeze dried 10\%

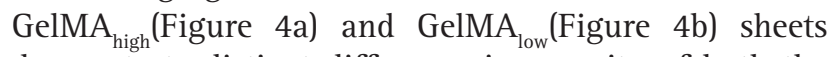
demonstrate distinct difference in porosity of both the gels. GelMA ${ }_{\text {low }}$ possesses visibly much larger pores as compared to GelMA $A_{\text {high }}$.

\section{Swelling analysis}

The degree of swelling of hydrogelsrelies on the porosity of the polymer. Change in mass swelling ratio of GelMA with respect toits concentrationand its methacrylation level was studied. 5\%, 10\%and20\% (w/v) GelMA $A_{\text {high }}$ and GelMA $_{\text {low }}$ were compared in terms of their swelling properties (Figure 4c). At any given concentration of GelMA, the mass swelling ratioof GelMA ${ }_{\text {low }}$ was significantly greater than that of GelMA high On the other hand, at a given degree of methacrylation, mass swelling ratio was inversely dependent on the concentration of GelMA.

Hoch et al. have demonstrated that the average size of pores in the scaffold matrix decreases with higher degrees of methacrylation and the degree of methacrylation was found to affect the physical and mechanical properties of the synthesized GelMA hydrogels, with higher methacrylation resulting in stiffer and more durable hydrogels, with smaller pore sizes (2012). In accordance with these studies, we observed that GelMA ${ }_{\text {low }}$ demonstrated significantly greater swelling as compared to GelMA $_{\text {high }}$ at any given concentration of GelMA. Swelling was also found to increase with decrease in concentration of GelMA. The results of swelling analysis were also in accordance with the porosity observed by SEM imaging, with GelMA ${ }_{\text {low }}$ demonstrating larger pores in comparison with GelMA ${ }_{\text {high }}$.

\section{HEPG2 CULTURE IN GELMA}

Highest porosity and least stiffness for both degrees of methacrylation was observed in 5\% GelMA. There- 


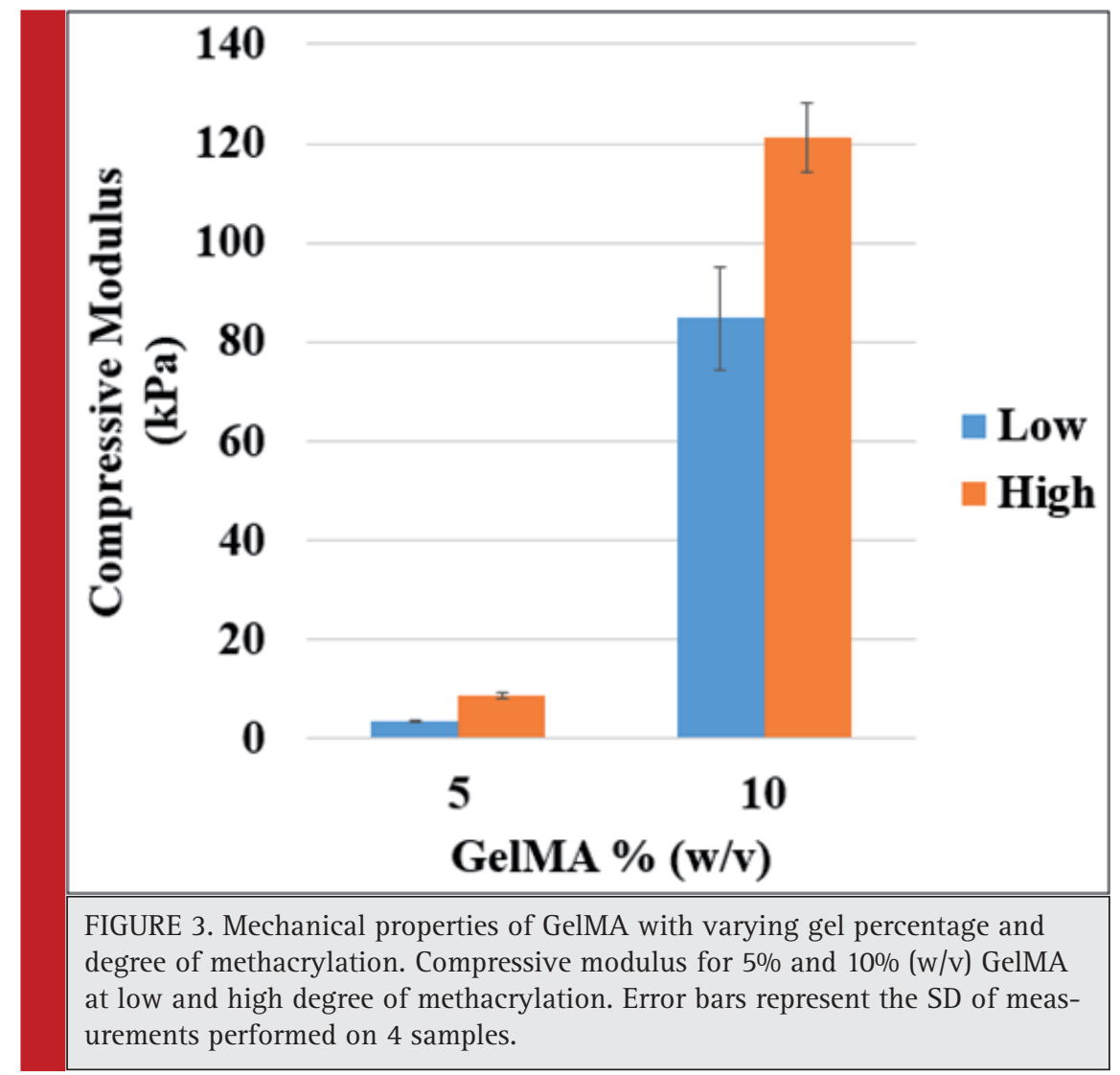

fore, taking into consideration intermittent stiffness and porosity, 10\% GelMA low $_{\text {andGelMA }}$ high were used for further encapsulation and cell culture study. HepG2 cells encapsulated in 10\% GelMA $\mathrm{high}_{\text {hin }}$ and GelMA ${ }_{\text {low }}$ were cultured for a span of 5 days in DMEM (Figure 5) so as to form $3 \mathrm{~d}$ sheet of $150 \mu \mathrm{m}$ thickness and was intermittently observed microscopically. Cells depicted morphology similar to what they demonstrate on $2 \mathrm{~d}$ culture. Therefore, encapsulation process, UV exposure and presence of photoinitiator didn't alter the morphology of HepG2 cells in GelMA.

\section{VIABILITY AND PROLIFERATION ANALYSIS}

\section{Live-dead staining analysis}

Viability of HepG2 cells encapsulated in GelMA ${ }_{\text {high }}$ (Figure 6a) and GelMA $A_{\text {low }}$ (Figure 6b) was observed microscopically on day 3 of culture by staining the sheets with calcein-ethidium homodimer. Live cells were stained green with calcein and dead cells appeared red stained with ethidium homodimer. Number of dead cells in Gel$\mathrm{MA}_{\text {high }}$ were visually much higher as compared to Gel$\mathrm{MA}_{\text {low }}$.Viability of cells in GelMA $\mathrm{l}_{\text {low }}$ were comparatively higher.

\section{MTT assay}

Proliferation of HepG2 cells cultured in 10\% GelMAhigh and GelMA $\mathrm{Aow}_{\text {low }}$ day 1, 3 and 5 was assessed using MTT assay (Figure 6c). In GelMA low , cells proliferated to achieve three times the number of cells from day 1 to day 5. On the contrary, some cells initially grew in Gel$\mathrm{MA}_{\text {high }}$, but failed to proliferate and demonstrated decline in cell number from day 1 to day 5. Therefore, GelMA ${ }_{\text {high }}$ proved to be hostile for culture of HepG2 cells as compared to GelMA low $_{\text {. On the other hand, GelMA }}$ proved to be much more favorable for HepG2 cell culture.

Cell viability has been shown to decrease with increasing polymer concentration (Hoch et al., 2012). Better proliferation was observed in GelMA ${ }_{\text {low }}$ as compared to GelMA $\mathrm{high}_{\text {as }}$ abserved via live-dead staining and MTT cell proliferation assay. The results were in line with the results previously reported by Occhettaet al. (2012). Nichol et al. also observed that in 5\% (w/v) gels, cell elongated, migrated and formed interconnected networks with neighboring cells, but not at higher concentrations of GelMA (2010). This explains that cells grow more effortlessly in softer gel matrices with large pores, where as in stiffer gel with smaller pore size, cells find it difficult proliferate and eventually die. 

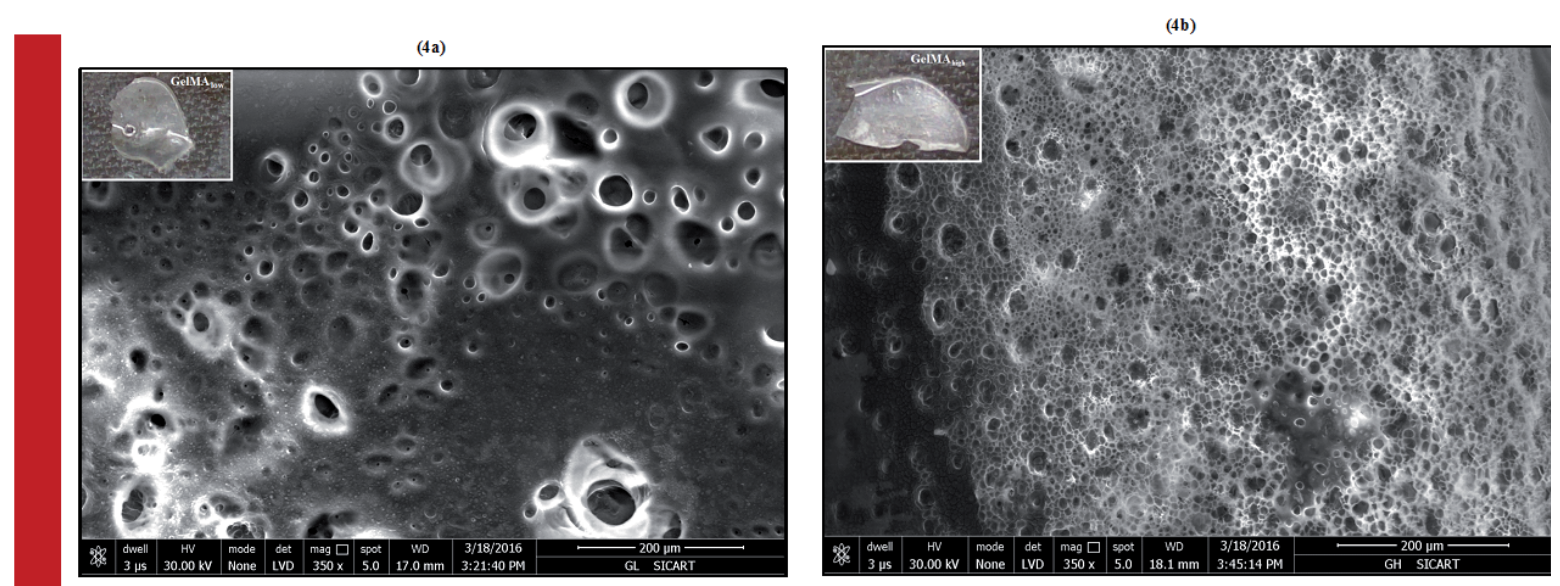

(4c)

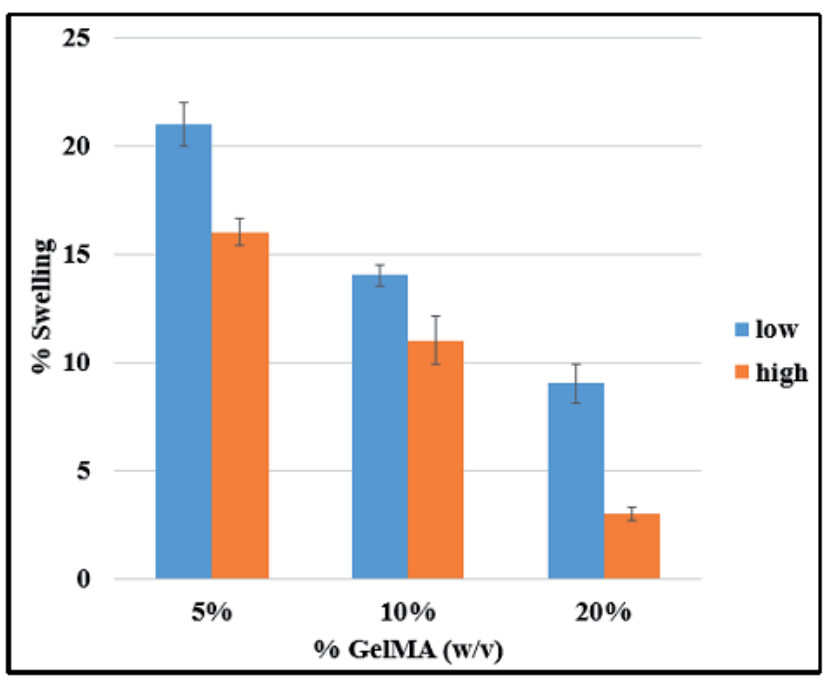

FIGURE 4. SEM images of freeze dried GelMA discs (a) low methacrylated and (b) high methacrylated. (Photographs of the discs used for imaging are on top left corner of images)(c) Swelling analysis of 5\% and 10\% of high and low methacrylated GelMA. Error bars represent the SD of measurements performed on 3 samples.

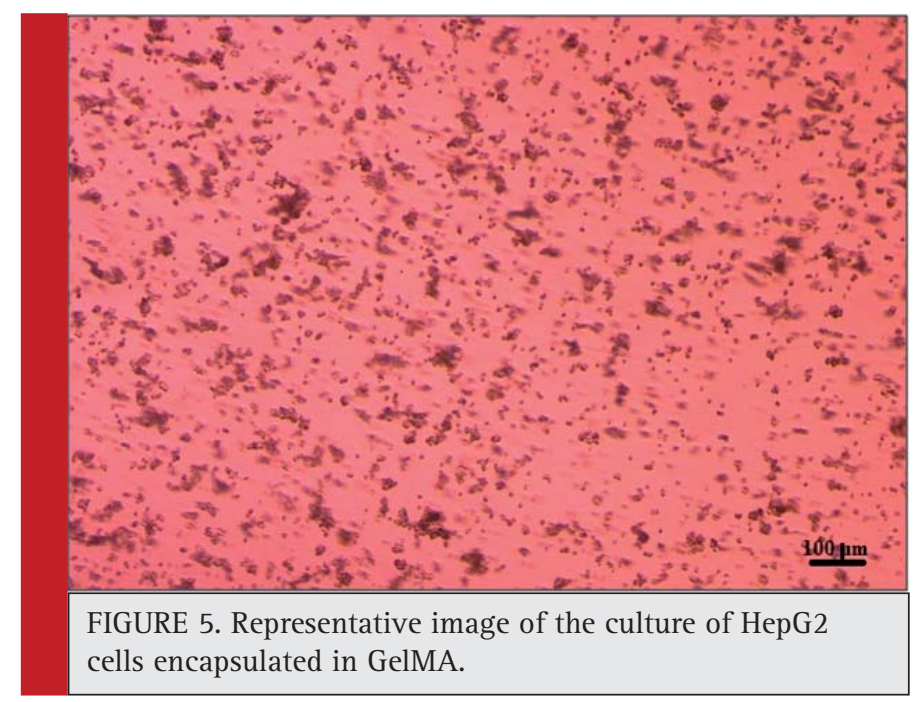



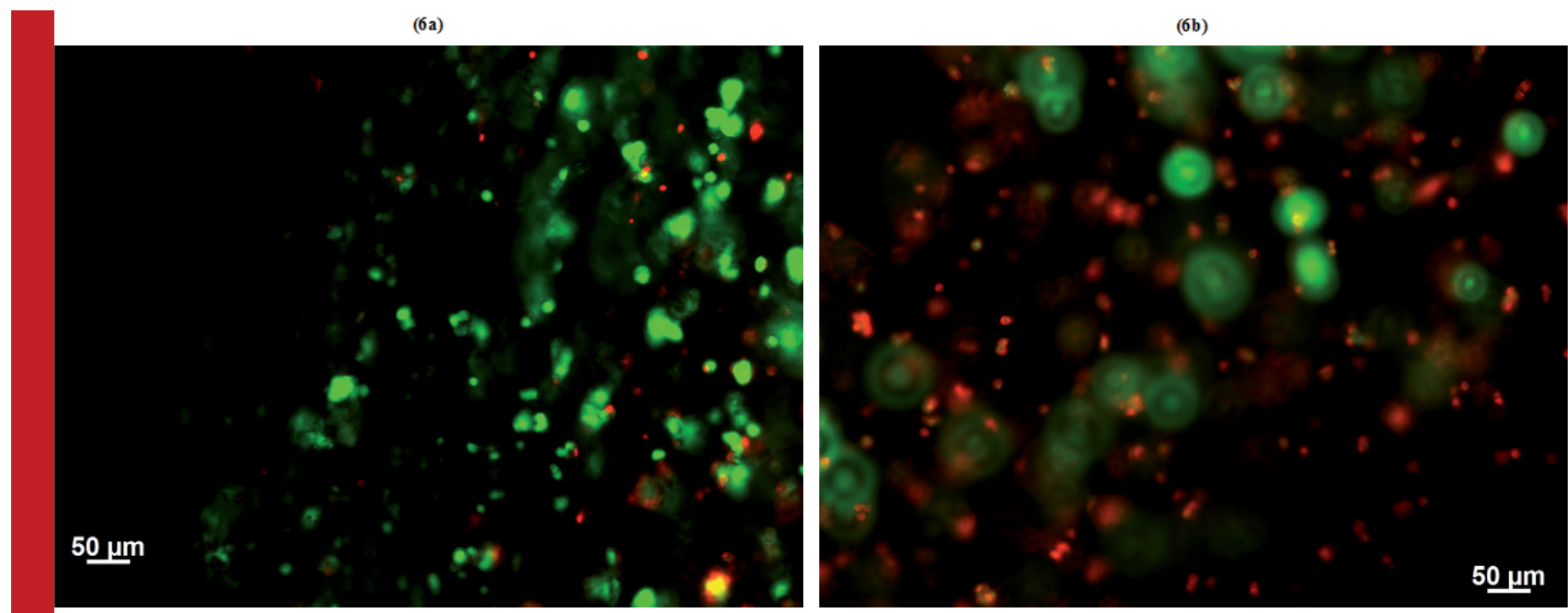

$(6 \mathrm{c})$

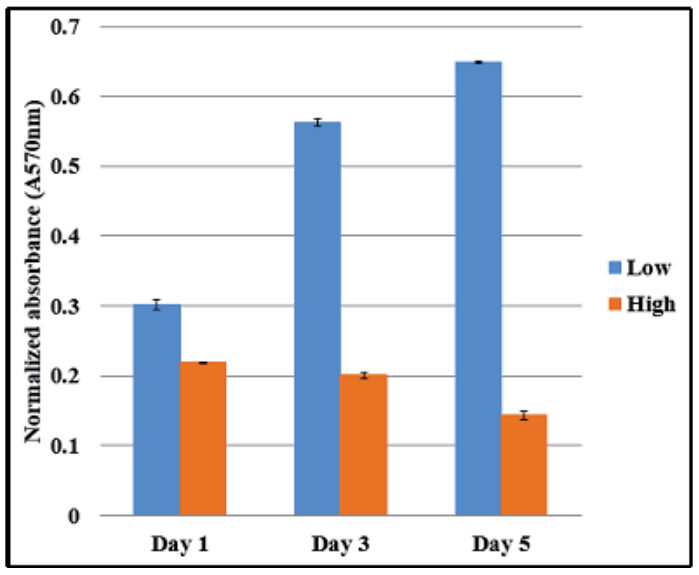

FIGURE 6. Representative images for Live-Dead staining of HepG2 cells on day 3 of culture encapsulated in (a) GelMA (b) GelMA high $_{\text {. }}$ (c) MTT assay for the proliferation of HepG2 cells encapsulated in GelMA $A_{\text {low }}$ and GelMA high $_{\text {measured on day }}$ 1, 3 and 5. Error bars represent the SD of measurements performed on 3 samples.

Therefore, present study puts forth the correlation between degree of methacrylation and concentration of GelMA with its porosity and stiffness. These very important physical matrix parameters can be fine-tuned and modulated as per the requirement and application for which this versatile biodegradable biocompatible hydrogel, GelMA, is intended to be used.

\section{ACKNOWLEDGEMENTS}

Authors are highly obliged to Dr. Ali Khademhosseini, Professor, Harvard University, Cambridge, USA for giving Mansi K. Aparnathi an opportunity to learn working with GelMA as a Pre-Doctoral Research Fellow in his esteemed laboratory at Harvard-MIT Health Science \&t Technology division. Authors are grateful to Anand Agricultural University, Anand, Gujarat, India for providing infrastruc- ture. Authors are thankful to Sophisticated Analytical Instruments Facility Supported by Department of Science \& Technology (Govt.of India), Vallabh Vidyanagar, Gujarat, India for facilitating SEM imaging.

\section{REFERENCES}

Basu S (2004) Effects of three dimensional structure of tissue scaffolds on animal cell culture. Ph.D. thesis, Ohio State University, Columbus, United States.

Bhatia S.N. and Chen C.S. (1999) Tissue Engineering at the Micro-Scale. Biomedical Microdevices Vol. 2 No. 2: Pages 131 144.

Chen Y.C., Lin R.Z., Qi H., Yang Y., Bae H., Melero-Martin J.M. andKhademhosseini A. (2012) Functional Human Vascular Network Generated in Photocrosslinkable Gelatin Methacrylate Hydrogels.Advanced Functional Materials Vol. 22 No. 10:Pages 2027-2039. 
Cretu A., Castagnino P. andAssoian R. (2010) Studying the Effects of Matrix Stiffness on Cellular Function using Acrylamide-based Hydrogels.JoVEVol. 42: Pages 2089.

Cuadros T.R.Erices A.A. and Aguilera J.M. (2015)Porous matrix of calcium alginate/gelatin with enhanced properties as scaffold for cell culture. J Mech Behav Biomed Mater Vol. 46: Pages 331-342.

Denizot F.and Lang R. (1986) Rapid colorimetric assay for cell growth and survival. Modifications to the tetrazolium dye procedure giving improved sensitivity and reliability. J Immunol Methods Vol. 89 No. 2.: Pages 271.

Du Y., Lo E., Ali S. and Khademhosseini A. (2008) Directed assembly of cell-laden microgels for fabrication of 3D tissue constructs. Proc Natl Acad Sci, Vol. 105 No. 28: Pages 9522-9527.

Hoch E.,Schuh C., Hirth T., Tovar G.E.M. andBorchers K. (2012) Stiff gelatin hydrogels can be photo-chemically synthesized from low viscous gelatin solutions using molecularly functionalized gelatin with a high degree of methacrylation.J. Mater. Sci.: Mater. Med. Vol. 23: Pages2607-2617.

Hollister S.J., Maddox R.D. and Taboas J.M. (2002) Optimal Design and fabrication of scaffolds to mimic tissue properties and satisfy biological constraints. Biomaterials Vol. 23: Pages 4095-4103.

Nichol J.W., Koshy S.T., Bae H., Hwang C.M.,Yamanlar S. and Khademhosseini A. (2010) Cell laden microengineered gelatin methacrylate hydrogels. BiomaterialsVol. 31: Pages 5536.

Nikkhah M., Eshak N., Zorlutuna P., Annabi N., Castello M., Kim K., Dolatshahi-Pirouz A., Edalat F., Bae H., Yang Y. and
Khademhosseini A. (2012) Directed Endothelial Cell Morphogenesis in Micropatterned Gelatin Methacrylate Hydrogels. Biomaterials Vol. 33 No. 35: Pages 9009-9018.

Occhetta P., Visone R., Russo L., Cipolla L., Moretti M. andRasponi M. (2014) VA-086 methacrylate gelatine photopolymerizable hydrogels: A parametric study for highly biocompatible 3D cell embedding. J Biomed Mater Res Part AVol. 00No. A:Pages 000-000.

Peppas N., Hilt J., Khademhosseini A. and Langer R. (2006) Hydrogels in biology and medicine: From molecular principles to bionanotechnology. Adv. Mater. Vol. 18 No. 11: Pages 1345-1360.

Samorezov J.E., Headley E.B., Everett C.R. andAlsberg E. (2016) Sustained presentation of BMP-2 enhances osteogenic differentiation of human adipose-derived stem cells in gelatin hydrogels.J Biomed Mater Res A (in press).

Tse J.R. and Engler A.J. (2010) Preparation of hydrogel substrates with tunable mechanical properties. Currentprotocols in cell biology. Chapter 10, Unit 10.16.

Tsuruga E., Takita H., Itoh H., Wakisaka Y. andKuboki Y. (1997) Pore size of porous hydroxyapatite as the cell-substratum controls BMP-induced osteogenesis. J Biochem Vol.121 No. 2: Pages317-324.

Van Den Bulcke Al., Bogdanov B., De Rooze N., Schacht E.H., Cornelissen M. andBerghmans H. (2000)Structural and rheological properties of methacrylamide modified gelatin hydrogels.Biomacromolecules Vol. 1: Pages 31-38. 